\title{
Diritti individuali e processo penale nell'Italia repubblicana
}

\author{
Direitos individuais e processo \\ penal na Itália republicana
}

\author{
Individual rights and criminal \\ procedure in republican Italy
}

\section{Renzo Orlandi}

Prof. ord. di dir. proc. penale - Alma Mater Studiorum - Università di Bologna renzo.orlandi@unibo.it.

Resumo: Com o presente artigo se propõe uma busca de diálogo interdisciplinar, entre cultores do direito positivo e históricos ou filósofos do direito, sobre as transformações do direito processual penal italiano e também acerca do papel que neste obtiveram os direitos fundamentais na Itália republicana. De 1946 em diante, as novas visões do relacionamento entre o indivíduo e o estado, entre liberdade e autoridade, entre garantia da pessoa e poder punitivo dos aparados públicos, configuraram em modo sensível e não sempre progressista o tipo de rito penal.

Palavras-chave: Direito Comparado. Processo Penal Italiano. Direito Individual.

ABSTRACT: This article proposes a search for the interdisciplinary dialogue between positive right and historical or philosophers of law, about the transformation of Italian Criminal Procedure LAw and also about the role that the fundamental rights had in the republican Italy. From 1946 onwards, the visions of the relationship between the individual and the state, between freedom and authority, between individual guarantees and punitive power of the State, configured in sensitive method and not always a progressive type of criminal rite.

KEY-wORDs: Comparative Law. Italian Criminal Procedure. Individual Rights. 
1. Premessa. La letteratura penalistica è ancora priva di una riflessione sulla storia recente del nostro diritto processuale. Una riflessione utilissima per il giurista positivo, tanto più in una fase di grande instabilità normativa come l'attuale.

Molte monografie (degli anni 60 e 70) informano sugli istituti di volta in volta considerati con resoconti stilati sulla scorta delle codificazioni precedenti (del 1865, del 1913). Le monografie successive alla riforma del 1988 appaiono meno orientate alla ricostruzione storica degli istituti. L'abbandono del modello processuale cosiddetto misto ha creato uno stacco rispetto alla tradizione precedente, sicché si reputa inutile mettere a confronto istituti considerati privi di una reale base di comparabilità. Francamente non è un male, considerato il carattere manierato, meramente compilativo e spesso superficiale di molte fra le ricostruzioni fornite dagli autori dei decenni passati. Del resto, non ci si può improvvisare storici. E, quasi sempre, il giurista positivo che si esercita nella comparazione diacronica lo fa da dilettante.

Ben vengano, dunque, le iniziative degli storici sul passato recente della nostra procedura penale. Sono l'occasione per un dialogo molto istruttivo. Penso, ad esempio, a Mario Sbriccoli e ai suoi studi sulla giustizia penale durante il fascismo ${ }^{1}$; a Marco Miletti, autore di una bella monografia sul codice del 1913²; penso ai saggi raccolti nel volume curato da Loredana Garlati sull'eredità del codice Rocco ${ }^{3}$ nonché, per il periodo a noi più vicino, ai saggi di Mario $\mathrm{Pisani}^{4}$ e di Ennio Amodio ${ }^{5}$.

1 Si veda, in particolare, il saggio Le mani in pasta e gli occhi al cielo, la penalistica italiana negli anni del fascismo, in Quaderni fiorentini, vol. XXVIII, Continuità e trasformazione: la scienza giuridica italiana tra fascismo e repubblica, 817 ss.

2 Un processo per la terza Italia: il Codice di procedura penale del 1913, Giuffrè, 2003.

3 L'inconscio inquisitorio, Giuffrè, 2010.

4 Autore dello scritto intitolato Il lungo cammino del codice 1988, inserito quale primo capitolo nel Manuale di procedura penale edito da Monduzzi, Bologna 2008 (VIII ed.).

5 Autore di uno scritto intitolato Processo penale, dedicato alla riforma del 1988 e pubblicato in Giuristi e legislatori. Pensiero giuridico e innovazione legislativa nel processo di produzione del diritto, $50^{\circ}$ volume dei Quaderni fiorentini per la storia del pensiero giuridico moderno, a cura di Paolo Grossi, Milano 1997, p. 363 ss. 
Segue questa scia l'idea lanciata dagli amici ferraresi di ripercorrere la storia delle riforme processuali nell'Italia repubblicana, con la tutela dei diritti individuali a far da filo conduttore. Una storia lunga fatta di slanci ideali e proiezioni ottimistiche nel futuro, ma anche di misoneismi e conseguenti impulsi di ritorno al passato. Comunque sia, una storia istruttiva per lo studioso, se non altro perché permette di evidenziare talune costanti della crisi che ha caratterizzato e caratterizza l'esperienza giuridica italiana nel settore della giustizia penale. Certo, non è una gran consolazione apprendere che, già negli anni ' 50 del secolo scorso, si lamentava l'eccessiva durata dei processi, si denunciava il ruolo marginale della difesa specialmente nella fase istruttoria, si segnalava l'insufficiente tutela della libertà personale. E' però di grande interesse ripercorrere gli argomenti esibiti nelle polemiche di quegli anni; soffermarsi sugli sfondi politico-sociali che hanno fatto da scenografia ai progetti di modifica della procedura penale dalla metà degli anni '40; meditare sul senso di fallimento che l'attuazione di ogni grande riforma prima o poi produce.

Le principali riviste penali offrono abbondante materiale per la nostra riflessione. Materiale poco studiato, soprattutto con riguardo agli anni' 40 e '50. Eppure è proprio nel periodo successivo alla caduta del fascismo e nell'immediato dopoguerra che il tema della riforma processuale penale è per la prima volta affrontato con le speranze e le paure tipiche dei momenti di grande svolta storica. Il lasso temporale che va dal 1944 al 1955 (anno della prima grande riforma processuale) merita di essere riconsiderato come il periodo nel quale si gettano le basi ideali delle riforme processuali penali.

Dagli anni '60 in poi la storia è assai meno misteriosa, ma merita anch'essa di essere raccontata, non foss'altro a vantaggio dei giovani che si avvicinano allo studio del diritto processuale penale. Ai meno giovani bastano pochi cenni per evocare le tappe di questo lungo e, per molti versi, appassionante itinerario; il "progetto Carnelutti" del 1962, preceduto dal convegno veneziano del settembre $1961^{6}$ il suo impatto

6 Intitolato ai Primi problemi della riforma del processo penale. Gli atti sono raccolti nel volume omonimo pubblicato a cura di G. De Luca, nei Quaderni di S. Giorgio, dall'editore Sansoni, Firenze 1962. 
nel dibattito sulla riforma del processo penale; lo storico convegno del 1964, svoltosi in due tempi fra Lecce e Bellagio; le riforme di stampo garantista propiziate da una coraggiosa giurisprudenza costituzionale che seppe essere segnavia per il legislatore. E poi la legislazione dei due decenni successivi: quella segnata dall'emergenza antiterrorismo degli anni '70 e quella, ad inizio degli anni ' 80, contrassegnata dall'emergenza antimafia, ma anche da una grave caduta di legittimazione della magistratura (soprattutto a seguito del caso Tortora) fino alla riforma processuale del 1988.

Quanto ai due decenni che ci separano dall'entrata in vigore del "nuovo" codice, la memoria è sin troppo fresca, al punto che farne la storia è impresa ardua, per eccesso di vicinanza all'oggetto osservato. Tuttavia, le involuzioni e i tentativi di controriforma, seguiti da aperture garantiste, in breve, le vicissitudini normative tortuose e contraddittorie, che hanno caratterizzato sin qui la vigenza del codice 1988, permettono di abbozzare una periodizzazione utile per individuare le linee di tendenza di una riforma processuale che è andata assumendo carattere permanente.

Il tema dei diritti individuali può essere affrontato in termini per così dire obiettivi, alla luce del principio di proporzionalità, al fine di stabilire fin dove possa spingersi l'esercizio di poteri coercitivi per ragioni di giustizia. Oppure può essere declinato in termini personalistici, sul piano dei poteri delle facoltà, dei diritti spettanti ai soggetti processuali. E' quest'ultimo, come si vedrà alla fine del nostro excursus, l'approccio prevalente nella dottrina italiana. Gran parte delle discussioni ruotano attorno allo spazio da riservare alla funzione difensiva e al correlativo contenimento della funzione accusatoria. Il diritto inviolabile di difesa, così come enunciato nell'art. 24 comma 2 cost., assorbe in sé o, meglio, oscura altri diritti individuali di cui possono esser titolari persone non coinvolte come parti nel processo (persone offese dal reato o persone coinvolte casualmente e indirettamente nell'attività investigativa e di accertamento).

2. Una proposta di periodizzazione. - E' impossibile abbracciare con uno sguardo un periodo lungo quasi settant'anni, né è consigliabile 
perdersi nei dettagli delle molte discussioni e iniziative che sul terreno della riforma processuale penale si sono susseguite dalla nascita della Repubblica italiana. Riesce meno complicata l'analisi e più comprensibile l'esposizione se questo lungo arco viene ripartito in segmenti temporali, scanditi da significative uniformità.

Ritengo adeguata ai nostri fini una divisione in tre parti del periodo considerato. La prima va dalla caduta del fascismo alla fine degli anni Cinquanta. La seconda, dall'inizio degli anni Sessanta alla riforma processuale maturata sul finire degli anni Ottanta. La terza, da codesta riforma ai giorni nostri.

La suggerita periodizzazione si fonda su un triplice distinguo: la situazione politico-sociale; lo stile dottrinale; la conseguente impostazione riformista.

Il primo periodo (1944-1961) inizia con l'Italia ancora in guerra: un Paese stordito dalla caduta del regime fascista e dalla catastrofe bellica, pronto però a investire energie nella ricostruzione sia materiale sia istituzionale. Anche il sistema penale di allora, a guardarlo dal nostro punto di vista odierno, appare come un edificio pericolante: da abbattere e ricostruire oppure da ristrutturare. Lo stile dottrinale è quello tecnico-giuridico, tipico anche dell'epoca fascista. Gli autori maggiormente presenti nel dibattitro e che più esercitavano influenza hanno i nomi di Giovanni Leone, Remo Pannain, Giuseppe Sabatini, Alfredo De Marsico. Sul piano delle iniziative e dei progetti, due sono le proposte che si fronteggiano: la prima, in ordine di tempo, ravvisando nel codice di rito del 1930 l'espressione del regime caduto, lo vorrebbe sostituire con il codice liberale del 1913; l'altra proposta, assai meno radicale, punta su una riforma parzialme del codice Rocco, sì da adeguarlo al nuovo clima democratico.

Il secondo periodo (1962-1989) ha inizio nell'Italia del boom economico. Un'Italia che, anche con riguardo alla riforma penale, coltiva un disegno utopistico: superare il modello processuale misto, ispirato al code d'instruction criminelle, sostituendo ad esso un modello adversary. Il 1962 è l'anno della svolta. Nel mese di gennaio il ministro della giustizia Gonella affida, all'ultraottantenne Francesco Carnelutti, il compito di progettare un nuovo codice di procedura penale. L'incarico coincide con 
un cambio generazionale. Attorno al vecchio Carnelutti si raccolgono giuristi quali Bellavista, Conso, Cordero, Delitala, De Luca, Foschini, Nuvolone, Sabatini e Vassalli: autori che - forse con le sole eccezioni di Giuseppe Sabatini e Giuliano Vassalli - non avevano partecipato alle diatribe del quindicennio precedente: quindicennio indubbiamente dominato dalla figura di Giovanni Leone. Nemmeno Carnelutti aveva svolto un ruolo importante in quelle discussioni, almeno a giudicare dal dibattito registrato sulle principali riviste penali degli anni Quaranta e Cinquanta ${ }^{7}$. E' questo un periodo che, sulla spinta del potente messaggio esistenzialistico e antiformalistico dell'ultimo Carnelutti, si apre all'idea di una riforma radicale del codice di rito penale. Il messaggio sarà raccolto dai giovani autori dell'epoca, in particolare da Franco Cordero, che diventerà un alfiere della riforma processuale in senso adversary. I1 periodo degli anni Sessanta si rivelerà ricco di progetti avveniristici.

Siamo in una fase di rapide e profonde trasformazioni sociali. L'Italia del boom economico è anche quella delle migrazioni interne da Sud a Nord. Si accentuano i processi di urbanizzazione ${ }^{8}$, con i disagi, le

7 Dalle ricerche effettuate sulle riviste dell'epoca risultano pressoché inesistenti le prese di posizione sulla riforma processuale penale di un giurista come lui, tanto incline a prender la parola su tutti i temi del momento e che, si badi, nella parte finale del suo insegnamento universitario (1946), all'età di sessantesette anni, si era "convertito" al diritto processuale penale. Unico intervento di questo periodo riguardante la riforma processuale penale è quello apparso su Giust. Pen. del 1945 (L'équità nelò giudizio penale, III, c. 1 ss.) dove si auspica una riforma delle Corti d'assise che restituisca alla giuria popolare la competenza a pronunciarsi sulla colpevolezza dell'imputato, sia pur con un pronunciamento dall'effetto soltanto consultivo (non vincolante) per il giudice togato. Una proposta destinata a cadere nel vuoto. Il nome di Carnelutti è pressoché assente anche dai numerosi convegni che fra la fine degli anni Quaranta e nel decennio successivo saranno dedicati alla riforma del processo penale. Il suo primo intervento significativo sul tema della riforma processuale risale al 1958 nel saggio Crisi della giustizia penale, dedicato a Emilio Betti e pubblicato in Riv. dir. proc. 1958, p. 333 ss.

8 Si registra un forte inurbamento, soprattutto nelle città del Nord-Italia. Stando alle rilevazioni sui censimenti Torino passa da circa 700 mila abitanti nel 1951 a più di 1 milione nel $1961(+42 \%)$ a 1 milione e 200 nel 1971 (+ 70\%). Milano, da 1 milione e 200 mila nel 1951 passa a più di un milione e mezzo nel $1961(+25 \%)$ e più di 1 milione 700 mila nel $1971(+41 \%)$. 
tensioni sociali e le situazioni di anomia che ne derivano. La tradizione rurale lascia il posto alla moderna cultura dei poli industriali. L'Italia dovrà fare i conti con forme di criminalità inedite e insidiose: aumento della criminalità predatoria, traffico di droga, sequestri di persona, terrorismo interno. Le prassi tradizionali, scarsamente incise dalla "riformetta del 1955" (come la chiamava Carnelutti), ispirate a regole farraginose e prive di sistematicità, appaiono inadeguate sia a fronteggiare la crescita di manifestazioni criminose tipiche della società del benessere, sia a garantire con la dovuta effettività la difesa dell'imputato.

Se si guarda alle iniziative assunte sul terreno della riforma processuale, si constata che nel periodo dal 1962 al 1988 numerosi progetti di riforma sono presentati in parlamento. Il dibattito nella comunità scientifica e fra gli operatori è intenso quanto appassionato. Le proposte cambiano nel tempo, sono diverse e spesso confliggenti fra loro, ma hanno in comune l'idea della riforma radicale e organica del processo: il codice Rocco non va revisionato in alcune parti; va sostituito non già col codice del 1913, con un codice nuovo, adeguato alla Costituzione e all'altezza dei tempi moderni. La dottrina favorevole a questo progetto è rappresentata principalmente dagli autori che avevano partecipato alla breve esperienza della Commissione Carnelutti. Vi si aggiungeranno, più tardi, studiosi come Giandomenico Pisapia e Delfino Siracusano, che avranno un ruolo importante nei passaggi successivi della riforma.

Nel frattempo, fra la fine degli anni Sessanta e gli anni Settanta, matura una nuova generazione di giuristi, cresciuta con la giurisprudenza della nostra Corte costituzionale e con una chiara consapevolezza del valore particolarissimo che spetta alle norme volte a tutelare diritti fondamentali; una generazione ormai interamente conquistata - per questa via - ai valori della costituzione repubblicana.

Sull'esempio di qualche lungimirante autore attivo anche negli anni Cinquanta (penso soprattutto a Giuliano Vassalli), si sviluppa in questo periodo un cambio di stile nella letteratura processualpenalisti-

Bologna da 340 mila nel 1951 a quasi 450 mila nel 1961 (+ 30\%), a circa 500 mila nel $1971(+47 \%)$. Roma, da 1 milione e 600 mila nel 1951 a più di 2 milioni nel $1961(+25 \%)$ a quasi 2 milioni 800 mila nel $1971(+75 \%)$. Inoltre, fra il 1951 e il 1971, l'Italia passa da 47 milioni a 53 milioni (+13\%). 
$\mathrm{ca}^{9}$. Basterà qui ricordare gli studi monografici sulla libertà personale di Giuliano Amato, di Ennio Amodio sulla motivazione della sentenza penale; di Vittorio Grevi sul nemo tenetur se detegere, di Massimo Nobili sul principio del libero convincimento, di Mario Chiavario su processo e garanzie della persona alla luce della Convenzione europea dei diritti dell'uomo, di Giulio Illuminati sulla presunzione di innocenza, di Paolo Ferrua sul principio di oralità. . Questi lavori, tutti concepiti quando si discuteva e, per così dire, si "sognava" un nuovo modello processuale, hanno tratto linfa dalle tensioni riformistiche di quegli anni; al contempo hanno accompagnato e favorito la riscrittura del codice, fornendo il supporto teorico e ideale per le soluzioni che si andavano elaborando.

Il terzo periodo si apre nel 1989. Qui possiamo individuare una data precisa: il 24 ottobre, il primo giorno di vita del nuovo codice. Il primo codice dell'Italia repubblicana, come si è soliti ripetere con una frase divenuta una sorta di refrain. Esso entra in vigore un anno dopo la sua pubblicazione, in un'Italia politicamente instabile (fra il 1986 e il 1989 la guida del governo cambia per ben quattro volte), con una magistratura in forte crisi di legittimazione (le polemiche intorno al caso Tortora sfociate nel referendum del 1987 sulla responsabilità civile dei magistrati) in un contesto internazionale che incubava trasformazioni (la fine dell'Unione sovietica) destinate, di lì a poco, a riflettersi anche sulla situazione politica interna.

9 Nella mente di molti magistrati s'era già fatta largo l'idea di un'interpretazione della legge necessariamente orientata ai valori costituzionali, come conferma l'ordine del giorno approvato al termine del Convegno organizzato a Gardone nel 1965 dall'Associazione nazionale magistrati. In esso si ripudiava l'idea che l'esercizio della giurisdizione dovesse ridursi a "una attività puramente formalistica indifferente al contenuto e all'incidenza concreta della norma nella vita del Paese"; e concludeva affermando che "Il giudice, all'opposto, deve essere consapevole della portata politico-costituzionale della propria funzione di garanzia, così da assicurare, pur negli invalicabili confini della sua subordinazione alla legge, un'applicazione della norma conforme alle finalità fondamentali volute dalla Costituzione". Il testo completo della mozione è riportato nel volume L'ordinamento giudiziario, a cura di A. Pizzorusso, Bologna, 1974, p. 34, nt. 47. 
L'eccesso di vicinanza impedisce di cogliere in poche, sintetiche battute il senso di questo ventennio. Un ventennio già ricco di vicissitudini sul piano delle riforme processuali che attendono di essere strutturate e sistemate in racconto. Sappiamo come la riforma del 1988 abbia subìto ben presto un serio ridimensionamento. Dopo esser stata, nei decenni precedenti, paladina del garantismo, la Corte costituzionale, forse spinta da una sorta di ratio compensandi, con le note sentenze del febbraio e del maggio 1992si mostra sensibile alle voci lamentose che dalla magistratura (specie quella requirente) si levano a criticare il nuovo assetto normativo..

$\mathrm{Ne}$ scaturisce uno squilibrio tra gli agonisti del processo che acuisce in misura patologica i contrasti fra accusa e difesa: contrasti degenerati, verso la metà degli anni ' 90 , in aperta ostilità fra magistratura e classe forense.

Credo che la febbrile attività di riforma della riforma o - se si preferisce - di controriforma, l'incessante produzione di novelle che ritoccano aspetti settoriali del codice di rito trovi spiegazione, almeno in parte, nell'accennato squilibrio fra le parti processuali. Almeno in parte, giacché - a partire dagli anni '90 - pure le legislazioni processuali di ordinamenti non caratterizzati da analoghe tensioni (penso, ad esempio, a Francia, Spagna, Germania, Austria) sono andate assumendo l'aspetto di cantieri sempre aperti.

Altro tratto di questo periodo, peraltro logicamente connesso con l'accennata situazione di continua instabilità normativa (tratto, questo sì, comune all'esperienza di altri ordinamenti), è la crescita di peso del formante giurisprudenziale: la perdita di centralità della legge statale propiziata per un verso dal sindacato costituzionale sulle leggi e, per altro verso, dalla cessione di sovranità derivante dall'inserimento dell'Italia nell'attuale contesto internazionale, esalta il ruolo dell'interprete e, in definitiva, del giudice che applica la norma nel caso concreto.

Ciò si riflette sulla qualità e sullo stile della letteratura odierna, comprensibilmente alla rincorsa dell'ultimo orientamento giurisprudenziale e al commento dell'ultima novella legislativa, da interpretare alla luce degli insegnamenti della Corte costituzionale, della Corte di Strasburgo, della Corte di giustizia delle Comunità europee. Risiede qui, a mio avviso, la ragione dello slittamento della produzione lettera- 
ria verso temi settoriali, accentuamente specialistici e tendenzialmente compilativi. Rare o quasi inesistenti le opere con pretese di sistemazione concettuale, sul tipo di quelle offerte dalla dottrina degli anni '60 e '70. Eppure non mancherebbero spunti per rivisitazioni di temi classici o per riflettere su temi nuovi, quali quelli suggeriti dal valore crescente che per il nostro diritto interno vanno assumendo le decisioni dell'Unione europea così come le norme dei Patti e delle convenzioni internazionali alla luce della giurisprudenza delle Corti europee.

3. La riforma processuale nel primo periodo (1944-1961). Conviene far coincidere l'inizio del primo periodo con il varo di talune leggi processuali immediatamente successive alla caduta del fascismo. Due anni abbondanti ci separano dal referendum istituzionale dal quale nascerà la Repubblica italiana. La retrodatazione si giustifica, perché già in quel periodo la legge processuale subisce modifiche ispirate all'affermazione dei diritti inviolabili della persona.

L'Italia è in guerra. La parte settentrionale ancora occupata dai tedeschi. Lì si pensa addirittura a un giudice speciale (l'alta corte di giustizia $)^{10}$ per giudicare i capi del fascismo. Insomma, non proprio il clima ideale per riforme processuali di stampo liberale. Eppure risale al gennaio del 1944 il primo significativo intervento legislativo in tema di fermo di indiziati di reato ${ }^{11}$. Nell'agosto dello stesso anno vedono la luce disposizioni legislative di impronta garantista in tema di durata della "custodia preventiva" 12 . Nel successivo mese di settembre, il potere di archiviazione del pubblico ministero - espressione di una giustizia penale direttamente condizionata dall'esecutivo e assoggettata alla ragion politica - viene subordinato al controllo del giudice istruttore ${ }^{13}$; la difesa dell'imputato nel dibattimento viene meglio garantita ${ }^{14}$.

10 Istituita con il d. lgs. lgt. 13 settembre 1944, n. 198.

11 R.d.l. 20 gennaio 1944, n. 45 recante Norme relative al fermo di indiziati di reato e di individui pericolosi per l'ordine sociale e la sicurezza pubblica

12 Art. 6 d. lgs. lgt. 10 agosto 1944, n. 194.

${ }_{13}$ L'art. 6 d. lgs. lgt. 14 settembre 1944, n. 288 modifica l'art. 74 c.p.p. 1930.

${ }^{14}$ L'art. 7 modifica gli artt. 468 e 469 c.p.p. 1930, sopprimendo i limiti temporali originariamente previsti per le arringhe difensive. 
Si coglie in codesti provvedimenti una prima reazione alla cultura giuridica autoritaria del ventennio: si tratta di provvedimenti provvisori, concepiti però nella prospettiva di una riforma dell'intera legislazione penale, percepita allora come imminente oltre che doverosa. Il proposito è formulato expressis verbis nel d. lgs. lgt. del settembre 1944: "Fino a quando non siano pubblicati i nuovi codici penale e di procedura penale - si legge nell'art. 1 - sono apportate le modificazioni di cui agli artt. seguenti al codice penale e al codice di procedura penale".

Del resto, che la caduta del fascismo - per le circostanze nelle quali era avvenuta - comportasse il ripudio degli eccessi autoritari dei quali la giustizia penale era intimamente compenetrata è opinione diffusa anche presso giuristi di stampo conservatore. "Il problema della riforma dei codici pubblicati fra il 1930 e il 1942 si presentò come immediato atto impulsivo sin dal mattino del 26 luglio 1943”, scriveva Remo Pannain nel 1945. Anche se subito sotto aggiungeva "Quel giorno tutto era spiegabile e giustificato. Poi subentrò la freddezza e la riflessione e il problema cominciò ad essere discusso in maniera diversa"15.

In effetti, il primo impulso era stato quello di un ritorno al passato pre-fascista. La commissione istituita nel gennaio 1945 dal ministro Tupini si era espressa a maggioranza per un ritorno al codice del 1913 "con l'impegno di aggiornarlo"16. Le componenti accademica e giudiziaria, minoritarie, erano invece favorevoli al mantenimento della legislazione vigente, debitamente depurata delle spigolosità autoritarie che il fascismo vi aveva introdotto.

Fra gli avversari del tuffo all'indietro si distinse, in particolare G. Leone. Significativo l'incipit di un suo intervento risalente al 1945: "Questo scritto mira a dimostrare come, ad onta delle opposte apparenze, il tradizionale complesso di principi giuridico-penali, di assodata origine liberale, sia stato, durante il ventennio trascorso, energicamente e quasi universalmente difeso e tenuto fermo dai giuristi italiani"17.

15 Notizie e spunti sulla riforma dei codici penali, in Arch. pen. 1945, p. 5.

16 Così riferisce Pannain nello scritto citato alla nt. precedente, p. 57.

17 G. Leone, La scienza giuridico-penale nell'ultimo ventennio, in Arch. pen. 1945,23 . Dello stesso tenore gli interventi svolti dal medesimo autore in $L a$ giustizia del 22 gennaio 1945 e su Il Domani d'Italia, del 10 febbraio 1945. 
Una simile posizione, condivisa e difesa da altri autorevoli giuristi dell'epoca, come Remo Pannain ${ }^{18}$, Francesco Santoro Passarelli ${ }^{19}$, Piero Calamandrei ${ }^{20}$, Giuseppe Sabatini ${ }^{21}$, finirà con l'imporsi negli anni successivi. Nel Congresso nazionale giuridico-forense del 1947 dedicato proprio alla riforma del codice di procedura penale, Giovanni Leone traccia le linee sulle quali lavoreranno, negli anni seguenti, le commissioni ministeriali impegnate nella revisione delle leggi penali fasciste. L'idea è sempre quella di salvare il codice di rito del 1930, innestandovi le modifiche necessarie a renderlo compatibile con i postulati dello Stato democratico ${ }^{22}$. Leone propone mutamenti che toccano la struttura stessa del processo (abolire l'istruzione sommaria; garantire l'istruzione formale per la generalità dei processi; ampliare i casi di citazione diretta a giudizio; riformare in senso garantista la disciplina delle impugnazioni) e toccano altresì le funzioni dei soggetti processuali (il pubblico ministero va alleggerito dei poteri spettanti al giudice; il ruolo del difensore va rafforzato anche attribuendogli "una certa sfera di documentazione di avvenimenti a lui noti”; alla persona offesa va riconosciuto il diritto a un'azione penale sussidiaria rispetto a quella del pubblico ministero). Mutamenti pesanti e profondi, sui quali è difficile trovare l'intesa sia dei politici sia degli operatori. Per questo, il convegno si chiuderà con una lunga mozione in 45 punti, dove si auspicano le modifiche reputate indifferibili per un'adeguata tutela dei diritti individuali: spiccano, in particolare, il diritto del difensore a partecipare a taluni atti dell'istruzione formale; il ripri-

A conclusioni analoghe giungeva, con riguardo al diritto penale del fascismo, T. Delogu, L'elemento politico nel codice penale, in Arch. pen. 1945, 161 ss., dove si legge che “... le linee fondamentali del codice non sono inspirate a principi contrastanti con un regime di libertà, mentre le infiltrazioni politiche riguardano più che altro questioni di dettaglio o singole figure di reati." (p. 193).

La riforma della legislazione, in Annali della Facoltà giuridica di Camerino, 1944, 35 ss.

19 E' tempestiva la riforma?, in Il Domani d'Italia, 3 febbraio 1945.

20 Sulla riforma dei codici - La truffa delle etichette, in La Nuova Europa, 4 marzo 1945.

21 La riforma del codice di procedura penale, in Rassegna giuridica, n. 1, 1945.

22 Il testo della relazione è pubblicato nella rivista Arch. Penale 1947, 442 ss. col titolo Sulla riforma del Codice di Procedura Penale. 
stino delle nullità assolute, come argine ai possibili abusi dell'autorità giudiziaria; l'imposizione di termini perentori alla durata della custodia preventiva; la facoltà di impugnare i provvedimenti limitativi della libertà personale; l'abolizione dell'obbligo per il latitante di costituirsi in carcere quale presupposto di ammissibilità dell'impugnazione; l'obbligo del giudice di pronunciarsi sulle istanze delle parti private.

Sono, questi, i punti che caratterizzeranno i progetti di riforma coltivati dalle Commissioni ministeriali attive nei primi anni '50, nelle quali Giovanni Leone eserciterà la sua indiscussa leadership.

Tale il disegno di legge presentato dal ministro Zoli alla Camera dei deputati nella seduta del 13 novembre $1952^{23}$ : il primo fra i progetti di riforma processuale elaborato tenendo conto anche della costituzione repubblicana entrata in vigore quattro anni prima. Approvato da un solo ramo del Parlamento nello scorcio finale della legislatura (23 marzo 1953), il d.d.l. in questione viene ripresentato sul limine della successiva legislatura, con qualche emendamento favorevole alla difesa e alle parti ${ }^{24}$. Finalmente il progetto diventa legge nel giugno 1955. La legge 19 giugno 1955, n. 517, prima grossa riforma del nostro processo penale, entra in vigore a metà luglio dello stesso anno. Essa sostituisce ben centodiciotto articoli del codice di rito e ne introduce diciotto di nuovi. Ma, al di là dei numeri, la riforma del 1955 altera in modo preoccupante l'equilibrio fra individuo e autorità: così essa viene percepita persino nellopinione degli accademici.

L'estensione di garanzie difensive allarma i primi commentatori. Secondo Remo Pannain, "la legge che entra in vigore il 15 luglio, inspirata a principi demagogici di cattiva lega, trascura del tutto l'interesse dello Stato e si prodiga in inchini e riverenze nei confronti dei delinquenti" ${ }^{25}$. Alfredo De Marsico, pur compiacendosi delle accresciute garanzie difensive, si mostra preoccupato per il notevole aumento di attività giudiziaria che ne deriva e per le conseguenti disfunzioni nell'esercizio della giurisdizione ${ }^{26}$.

${ }^{23}$ La relativa relazione è pubblicata in Arch. penale 1952, 406.

${ }^{24}$ Come affermato dal ministro De Pietro nella relazione alla Camera dei deputati: il testo è in Riv. pen. 1954, 569 ss.

25 La riforma della procedura penale, in Arch. penale 1955, 371.

${ }^{26}$ Problemi della giustizia penale, ibidem, 482. 
Nell'aprile del 1956 comincia ad operare la Corte costituzionale, destinata ad avere un ruolo di primissimo piano nella tutela dei diritti individuali, in quella zona di confine per la loro tutela rappresentata dal diritto processuale penale ${ }^{27}$. E' meritevole di segnalazione la circostanza che, fra le 34 decisioni di quel primo anno d'attività, nessuna riguardi il codice di rito penale (molte sono riferite al testo unico di pubblica sicurezza; altre a norme penali sostanziali). Bisogna dunque riconoscere che al primo impatto con la concreta applicazione della carta costituzionali, la riforma maturata l'anno precedente rivela una sua indubbia capacità di tenuta.

Nella seconda metà degli anni ' 50 si parla meno di riforma processuale. Negli indici delle riviste il tema non occupa più lo spazio riservatogli nella prima metà del decennio. L'attenzione si rivolge ora alla riforma del diritto penale e dell'esecuzione penale ${ }^{28}$

Bisogna attendere qualche anno, prima che affiori, all'inizio del decennio successivo, l'insoddisfazione per l'eccessiva complicatezza della legge processuale novellata, nella quale si ravvisa la causa di intollerabili lungaggini. Il tema prevalente diventa, pertanto, la struttura del processo, le finalità delle singole fasi, la distribuzione di poteri e facoltà tra i soggetti processuali. Laccento si sposta dai diritti e relativa tutela alle funzioni processuali e relative situazioni soggettive.

A dar voce all'insoddisfazione è Francesco Carnelutti, in un convegno veneziano da lui stesso organizzato nel settembre $1961^{29}$.

Come accennato in precedenza, Carnelutti aveva praticamente disertato le discussioni e le diatribe dottrinali sfociate nella riforma del $1955^{30}$. Resta assai misteriosa la ragione che lo spingerà, già molto anzia-

27 La giurisprudenza costituzionale è, per anni, il vero motore della riforma processuale, considerata la difficoltà legislativa a realizzare una riforma globale, come osserva A. Malinverni, La riforma del processo penale, Torino 1970, p. 43.

28 Ad alcune fra le più urgenti riforme del diritto penale sono dedicati, ad esempio, i convegni nazionali di studio organizzati nel 1958 e nel 1959 dal Centro nazionale di prevenzione e difesa sociale.

${ }_{29}$ Si veda il resoconto che ne fa Giuseppe De Luca, in Riv. it. dir. e proc. pen. 1961, 1040.

30 Vedi tuttavia l'intervento al convegno dell'aprile 1953, dove Carnelutti (sorprendentemente, se si pensa all'iniziativa che intraprenderà circa 10 anni più tardi) invita a diffidare del modello accusatorio, "pericoloso perché siamo 
no, a dedicare le proprie energie alla riforma del processo penale. A spiegarla, almeno in parte, contribuisce la circostanza che Giovanni Leone, divenuto proprio nel 1955 presidente della Camera dei deputati, era stato costretto ad abbandonare il campo. Egli non poteva più esercitare - sul piano dottrinale - l'influenza avuta nelle discussioni degli anni preceden$\mathrm{ti}^{31}$. Nessuno, fra i giuristi dell'epoca, aveva osato o era stato in grado di occupare la posizione di preminenza che Leone si era conquistato. All'inizio degli anni'60 ci provò e ci riuscì Francesco Carnelutti, il quale - oltretutto - nutriva scarso apprezzamento verso il collega-rivale napoletano ${ }^{32}$.

\section{Secondo periodo: dal progetto Carnelutti all'entrata in vigore del} nuovo codice di procedura penale (1962-1989). -

Il convegno veneziano voluto da Carnelutti segna dunque una ripresa d'interesse sulla riforma processuale penale, pur limitata, per il momento, a pochi, selezionati interlocutori ${ }^{33}$. Qualche mese più tardi,

troppo intelligenti in Italia"; cfr. Atti del convegno fra le più urgenti riforme del c.p.p., Giuffrè, Milano 1954, 81.

31 Leone viene eletto presidente della Camera il 10 maggio 1955 e riceverà altri due mandati nelle legislature successive (fino al 15 maggio 1963).

32 Il difficile rapporto fra due grandi processualisti emerge con tutta evidenza nello scritto $A$ proposito di alcuni giudizi del prof. Carnelutti, pubblicato da Leone in Riv. it. dir. e proc. pen. 1962, 15 ss. Con toni caustici, Leone rimprovera il collega di aver recensito il suo Trattato di diritto processuale penale, senza nemmeno averlo letto. Ma la critica più severa (e amara) è riservata al modo sprezzante col quale Carnelutti, nella prefazione dei suoi Principi del processo penale (Napoli, Morano, 1960, 2), parlava di Leone come colui "che insegna, nientemeno, la nostra materia all'Università di Roma”.

Dal canto suo, Giovanni Leone giudicherà molto severamente lo sforzo riformistico di Carnelutti. Si veda quanto riferisce al riguardo M. Pisani, Variazioni in tema di <<bozza>> Carnelutti per un nuovo c.p.p., in Riv, dir. proc. 2010, p. 88, note 12 e 13.

33 Più che un convegno, un seminario condotto con la tecnica del brain-storming, al quale partecipano, oltre allo stesso Carnelutti, accademici (Bellavista, Conso, Cordero, De Luca, De Marsico, G. Foschini, C. Massa, Pannain, Petrocelli, Pisapia, Gius. Sabatini e Vassalli), alcuni dei quali anche nella veste di avvocati nonché magistrati locali e di Cassazione (Cabrini, Caprioglio, A. Foschini, Grieco, Lattanzi, Liguori, Loschiavo, Piacenza, Scandellari, Velotti, Vista e Zingale). 
nel gennaio del 1962, il ministro della Giustizia Gonella, presente a quel convegno, istituirà una commissione per la riforma del codice di rito penale e chiamerà proprio Carnelutti a presiederla ${ }^{34}$. Dominata dall'invadente personalità del presidente, quella commissione opererà con scarso senso della collegialità ${ }^{35}$. Dopo una prima riunione nel febbraio 1962, Carnelutti si impegna a redigere molto rapidamente uno "schema originale" che, riveduto dal magistrato di cassazione Nicola Reale e da Giuliano Vassalli, sarà poi ampiamente emendato e presentato alla Commissione nelle successive riunioni del maggio e del settembre 1962.

Il contesto politico-sociale non è più quello degli anni ' 50 . Diverso è inoltre l'approccio intellettuale al tema della riforma processuale. L'iniziativa di Carnelutti risulta molto innovativa rispetto alle discussioni degli anni precedenti, limitate - come sè visto - all'alternativa di un ritorno al passato (codice del 1913) ovvero di una riforma del codice esistente per adeguarlo ai principi della costituzione. Non aveva raccolto adepti l'idea di un nuovo codice di rito penale, reso necessario dal mutato clima politico nell'Italia repubblicana, conquistata ai valori democratici ${ }^{36}$.

Carnelutti muove dall'esigenza di un profondo cambiamento, benché nemmeno lui sembri preoccuparsi della ragione politica della

34 Il nesso fra il convegno veneziano e l'idea di una nuova commissione per la riforma processuale è attestato dall stesso Carnelutti nella Avvertenza al volumeVerso la riforma del processo penale, Napoli, Morano, 1963, 5, che riproduce il progetto da lui redatto.

Sulla commissione Carnelutti e sull'atmosfera che vi si respirava è di noteveole interesse l'eccezionale testimoninanza offerta dal prof. Giuliano Vassalli nell'Introduzione al volume L'inconscio inquisitorio, cit., 9 ss.

35 Come ammette lo stesso Carnelutti nell'Avvertenza citata alla nota precedente. L'esperienza di codesta Commissione è efficacemente ricostruita da O. Mazza, L'illusione accusatoria: Carnelutti e il modello dell'inchiesta preliminare di parte, in L'inconscio inquisitorio, cit. 153 ss

36 Lidea trova pochi fautori: fra questi, Giuseppe Guarneri, autore sottovalutato, docente di procedura penale all'Università di Parma, che nel saggio Osservazioni sul progetto di modificazioni per l'aggiornamento del Codice di procedura penale, in Arch. penale, 1950, 453 ss. denuncia il considerevole iato tra diritto vigente e diritto preannunciato dalla carta costituzionale e reputa del tutto insufficienti le iniziative fin lì discusse e intraprese per colmarlo. 
riforma. "Io sono convinto - scrive nell'Introduzione allo schema del suo progetto - che loperseguito in Italia negli ultimi trent'anni consenta, anzi esiga, una modificazione dei principii, sui quali è fondato il codice vigente, così profonda che non è possibile provvedere a un restauro del codice stesso, del quale è invece necessaria una coraggiosa ricostruzione”.

Composto di 227 articoli, lo schema carneluttiano è un punto di svolta nella storia della riforma processuale italiana. Esso si caratterizza soprattutto per la risistemazioine del procedimento di primo grado, dove si stabilisce una separazione netta tra "inchiesta preliminare del pubblico ministero" (finalizzata alla elaborazione dell'accusa) e "fase definitiva del processo di cognizione" (finalizzata alla decisione di merito). Si abbandona così il modello - ispirato al code d'instruction criminelle del 1808 - divenuto tradizionale anche in Italia grazie al suo affermarsi nelle tre codificazioni dell'Italia unitaria $(1865,1913$ e 1930).

Nelle parole dell'autore, l'esigenza di cambiamento ha radice intellettuale e teorica ("lo studio del diritto processuale") più che politico-costituzionale. A evidenziare ed enfatizzare il valore politico della rivoluzionaria proposta di riforma contribuirà poi, con efficaci argomenti, Franco Cordero, uno dei membri (il più giovane) della commissione Carnelutti. Loccasione è data dal convegno dedicato ai Criteri direttivi per una riforma del processo penale, organizzato dal centro nazionale di prevenzione e difesa sociale e destinato a svolgersi in due tempi: nel maggio 1964 a Lecce e nel successivo ottobre a Bellagio.

Nella mozione conclusiva si prende atto della difformità d'opinioni registrata intorno al modo di condurre la fase preliminare del processo. Tutti concordano sull'opportunità di abolire il dualismo istruttorio (all'istruzione formale, affidata al giudice istruttore, era giustapposta l'istruzione sommaria, affidata al pubblico ministero). Le opinioni differivano tuttavia con riguardo al modo per attuare la semplificazione. Tre orientamenti si dividevano il campo: (a) abolire l'istruzione sommaria e mantenere l'istruzione formale con adeguate garanzie per la difesa (proposta Pietro Nuvolone alla quale aderiva anche Giovanni Leone); (b) abolire l'istruzione formale e generalizzare l'istruzione sommaria, con le garanzie difensive imposte dall'art. 24 cost. (proposta dei magistrati Giuseppe Altavista e Girolamo Tartaglione 
appoggiata anche da Giovanni Conso); (c) introdurre l'inchiesta preliminare di matrice carneluttiana (proposta di Franco Cordero) ${ }^{37}$.

In quello stesso periodo riprendono, febbrili, iniziative di riforma sul terreno parlamentare. Lo schema di Carnelutti non si traduce in proposta legislativa. Si afferma però l'idea che il codice vada ricostruito, riscritto per intero, non soltanto revisionato in alcune sue parti. La pur ampia riforma del 1955 appare insufficiente ad assicurare la necessaria efficacia dell'azione repressiva e, al contempo, inadeguata ad attuare i principi della Costituzione con i diritti inviolabili dalla stessa riconosciuti. La riscrittura del codice consiglia una divisione del lavoro tra Parlamento e Governo: strumento adeguato a tal fine è la legge-delega, mai sperimentata fino all'inizio degli anni sessanta. La prima è del 1963 e riguarda tutti e quattro i codici. Ma solo la delega relativa al codice di procedura penale sarà coltivata negli anni successivi. Fra il 1963 e il 1974 si contano una diecina di deleghe proposte dal parlamento ai molti governi susseguitisi. Le tredici direttive elencate nel disegno di legge presentato alla camera dei deputati dal ministro Bosco il 10 ottobre $1963^{38}$ lievitano a 85 nella delega legislativa approvata finalmente in via definitiva dal Senato il 3 aprile $1974^{39}$.

Si afferma altresì l'idea che la riforma processuale sia essenzialmente una questione di equilibrio tra le forze in campo. In tutti i progetti di questo periodo si auspica l'attuazione di un sistema accusatorio, si persegue la parità fra le parti, si celebrano le garanzie difensive anche nella fase preliminare del processo, si esaltano il contraddittorio, l'oralità,

37 Per bravura di relatori ed efficacia di interventi, il convegno di LecceBellagio raggiunse vette qualitative forse non più eguagliate nei dibattiti (numerosissimi) sulla riforma processuale. Gli atti sono pubblicati a cura del Centro nazionale di prevenzione e difesa sociale nel volume Criteri direttivi per una riforma del processo penale, Giuffrè, Milano 1965. La mozione conclusiva è riportata alle p.p. 405-406.

Il testo, con la relazione, è pubblicato in Riv. it. dir. e proc. pen. 1963, 110 ss.

Legge 3 aprile 1974, n. 108. Testo e relazione in Riv. it. dir. e proc.pen. 1974, 400 ss. Una ricostruzione molto ben documentata degli atti parlamentari sfociati nella 1. delega del 1974 è offerta da G. Conso, Precedenti storici e iter della legge n. 108 del 1974, in G. Conso, V. Grevi, G. Neppi Modona, Il nuovo codice di procedura penale. Dalle leggi delega ai deccreti delegati, vol. I, La legge delega del 1974 e il progetto preliminare del 1978, Cedam, Padova 1989, 3 ss. 
la concentrazione nella fase di merito. Fra i diritti inviolabili riconosciuti dalla costituzione, lo spazio preponderante spetta senza dubbio al diritto di difesa. Espanderlo oltre i limiti assicurati dalla citata riforma del 1955 comporta la messa in discussione del sistema "misto" o "bifasico", che quella riforma aveva inteso preservare.

Proprio in questo rilievo risiede - a mio avviso - la ragione che ha spinto il legislatore a progettare un nuovo codice di procedura penale, anziché proseguire nella riforma di quello vigente. Proseguire sulla via del garantismo inquisitorio - per riprendere l'icastica espressione coniata da Ennio Amodio - significava accrescere l'importanza della fase istruttoria e, al contempo, aggravarne l'ipertrofia.

Anche la Corte costituzionale si muove lungo la linea di una progressiva affermazione del diritto di difesa nella fase investigativa. La sua giurisprudenza negli anni '60, permetterà di ampliare considerevolmente le garanzie di partecipazione del difensore nell'istruzione formale, in quella sommaria e persino nella cosiddetta fase preistruttoria condotta dalla polizia. Anche qui, la norma che ricorre più spesso quale parametro nelle valutazioni di illegittimità, è l'art. 24 comma 2, spesso combinato con l'art. 3 , al fine di censurare irragionevoli disparità di trattamento fra le posizioni dell'imputato nelle diverse fasi del procedimento o per ridurre la distanza fra difensore e pubblico ministero. Ben più sporadici gli interventi fondati su asseriti contrasti della legislazione ordinaria con altri diritti inviolabili quali libertà personale, libertà e segretezza di comunicazione e corrispondenza, presunzione d'innocenza. Anche nella mente dei giudici rimettenti si è dunque instillata l'idea che l'adeguamento ai valori costituzionali passa, essenzialmente, per un'attuazione del diritto di difesa e, in ultima analisi, per una modifica degli equlibri fra accusa e difesa. L'enfasi sulla garanzia difensiva costituisce un tratto tipico nell'evoluzione recente della nostra procedura penale; un tratto non riscontrabile, ad esempio, nella coeva e similare esperienza di altri ordinamenti come, ad esempio, quello germanico ${ }^{40}$.

40 Dove il Bundesverfassungsgericht, a partire dal 1952, ha per così dire bonificato la legislazione processuale facendo leva sul principio di proporzionalità (Verhältnismässigkeitsgrundsatz) alla luce dell'esigenza di tutelare la dignità (Menschenwürde: Art. 1 Grundgesetz) di tutte le persone a qualsiasi titolo coinvolte nel processo (non del solo imputato). 
La delega del 1974, nell'incipit dell'art. 1, "promette" un articolato definitivo del nuovo codice di procedura penale "entro due anni dall'entrata in vigore della presente legge". Redatto da una Commissione presieduta dal prof. Giandomenico Pisapia, l'articolato sarà pronto con due anni di ritardo, nel 1978, in un momento assai poco propizio per riforme dal sapore garantistico. Siamo in piena emergenza antiterrorismo. Un'emergenza che già da qualche anno aveva trovato riscontro sul piano delle iniziative legislative. E' curioso osservare che, a una settimana dall'approvazione della legge-delega per la riforma del processo, il Governo vara un decreto-legge contenente "Provvedimenti urgenti sulla giustizia penale" ${ }^{\text {11 }}$ per fronteggiare l'allarmante fenomeno dei sequestri di persona a scopo di estorsione: sarà la prima di una lunga serie di leggi d'emergenza approvate nel corso degli anni '70.

Eppure, l'inversione di tendenza non fa venir meno la tensione verso la riforma processuale. Accade che alcuni provvedimenti emergenziali facciano - per così dire - tesoro di talune proposte tradotte in direttive di legge-delega o in articoli elaborati e discussi dalla commissione Pisapia. E' il caso, ad esempio, della 1. 152 del 1975 (la cosiddetta legge Reale, una delle più controverse leggi d'emergenza) che, traendo spunto da una direttiva della delega 1974, riconosce alla "tutela della collettivită" il rango di possibile esigenza cautelare ${ }^{42}$ con anticipo sulla riforma processuale $^{43}$. E un discorso analogo vale per la 1.534 del 1977 (Modificazioni al codice di procedura penale) che modifica la disciplina delle nullità nel senso previsto dalla Commissione Pisapia. Insomma, il periodo emergenziale non fa tramontare il disegno di riforma processuale, anche se nel 1979, il ministro della giustizia (Morlino) - come ricorda Vassalli - "sospese i lavori a causa delle modifiche sopravvenute nella legislazione speciale"44.

41 D.1. 11 aprile 1974, n. 99 (conv. nella 1.7 giugno 1974, n. 220).

${ }^{42}$ Precisamente la dir. nr. 54 dell'art. 2 1. delega 1974. Il particolare è rilevato da Corte cost. 1/1980, che dichiara parzialmente illegittimo l'art. 1 della citata 1.152/1975.

43 Oltretutto, la natura eccezionale e temporanea della stessa "legge Reale" è ben presente alla consapevolezza dei compilatori, quando scrivono, nell'art. 35, che "le disposizioni della presente legge si applicano sino all'entrata in vigore del nuovo codice di procedura penale".

44 Introduzione, cit., 12. 
Oggi possiamo dire che il momento per l'approvazione del nuovo codice fu solo rinviato di qualche anno. I lavori riprenderanno verso la fine del 1983, finita l'emergenza terroristica, per iniziativa del ministro della giustizia Martinazzoli, il quale istituirà una commissione presieduta di nuovo dal prof. Pisapia, in segno di continuità con l'esperienza maturata sulla legge-delega del 1974 e sfociata nel ricordato progetto preliminare del 1978.

La nuova delega, ricca di ben 105 direttive, è pronta nel febbraio $1987^{45}$ e meno di un anno sarà sufficiente per redigere il corrispondente progetto preliminare di nuovo codice ${ }^{46}$. A fine settembre del 1988 è pronta la versione definitiva del nuovo codice, pubblicata poi il 24 ottobre su un supplemento ordinario della Gazzetta ufficiale.

La rapidità di redazione si spiega col fatto che la commissione Pisapia attinge largamente ai risultati del decennio precedente. Ma ciò non spiega tutto. Credo che il varo del nuovo codice sarebbe stato assai più difficile e faticoso, se in quel periodo - come accennato in precedenza - la magistratura italiana non fosse precipatata in una forte crisi di legittimazione. Le discussioni e le polemiche seguite al caso Tortora (1983) e la conseguente iniziativa referendaria sulla responsabilità civile dei magistrati (1987) prepararono, a mio avviso, il terreno per attuare quella radicale riforma processuale della quale si parlava da quasi trent'anni senza esser capaci di tradurla in norma effettiva.

Entrato in vigore il 24 ottobre 1989, il nuovo codice di rito penale rivela una notevole affinità con l'idea carneluttiana dell'inchiesta di parte. L'idea-guida è quella di una marcata separazione tra fase preliminare e fase del giudizio. L'attuazione del diritto di difendersi,è perseguita attraverso un riequilibrio nei rapporti di forza fra accusa e difesa. Il pubblico ministero è signore incontrastato dell'indagine preliminare, ma le conoscenze da lui acquisite servono, di regola, al solo fine di preparare l'accusa e poi sostenerla con prove e buoni argomenti davanti al

45 Approvata con 1.16 febbraio 1987, n. 81.

${ }^{46}$ In data 29 gennaio 1988 il testo del progetto preliminare viene trasmesso dal consiglio dei ministri ai due rami del parlamento per l'esame da parte della commissione incaricata di esprimere il parere di conformità alle direttive della legge delega. 
giudice del dibattimento. La separazione tra fase preliminare e fase di merito cambia i rapporti fra i soggetti processuali in quel campo di forze che è l'agone processuale. Ben presto si capirà che il nuovo equilibrio faticherà ad affermarsi a causa delle resistenze inocntrate all'interno dell'ordine giudiziario.

5. Terzo periodo: l'esperienza del primo codice dell'Italia Repubblicana (1989-2010).-

Più di vent'anni ci separano dall'entrata in vigore del primo codice dell'Italia repubblicana. Un tempo che offre abbondante materiale di riflessione nella prospettiva che qui interessa, quella della riforma processuale attuate sotto il segno dei diritti individuali.

Il contesto politico-sociale è segnato dalla fine della guerra fredda e dalla successiva globalizzazione dei mercati, che trascina con sé trasformazioni atte a incidere profondamente sulla sovranità dello Stato e, quindi, sulla qualità delle fonti normative ${ }^{47}$.

Quanto allo stile dottrinale, è difficile individuarne uno che appaia oggi prevalente negli studi processualpenalistici. Ancora una volta, l'eccesso di vicinanza nuoce. Si può comunque rilevare come l'entrata in vigore del nuovo codice e le numerose modifiche che ne sono seguite abbiano imposto agli studiosi una frenetica attività di commento e aggiornamento a carattere essenzialmente esegetico, lasciando poco tempo per l'analisi o la rivisitazione sistematica degli istituti. Inoltre, s'è persa quella tensione verso il nuovo che caratterizzò i tre decenni precedenti la riforma del 1988 e che ispirò i migliori saggi monografici di quel periodo. La diagnosi dell'odierno stile dottrinale appare difficoltosa, ma c'è spa-

47 Il fenomeno della progressiva perdita di pregnanza del diritto statuale nell'era della globalizzazione riguarda ogni settore dell'esperienza giuridica, non escluso, ovviamente, il campo penale, così esposto ai venti della politica. Per istruttive riflessioni sulla portata del fenomeno si vedano gli studi di M. R. Ferrarese, La governance tra politica e diritto, Bologna 2010, alla quale si rinvia anche per la ricca bibliografia sui riflessi che la globalizzazione economica è capace di irradiare sulle istituzioni pubbliche nazionali. Si vedano, inoltre, sul declino dello Stato nazionale S. Cassese, La crisi dello Stato, Roma 2002 e P. Grossi, Globalizzazione, diritto, scienza giuridica, in Società, diritto, Stato, Milano 2006, p. 279 ss. 
zio almeno per un auspicio. Un ritorno al tecnicismo del primo periodo è improponibile, essendo ancora attuali le ragioni che indusssero molti autori negli anni '60-'70 del secolo scorso ad abbandonare tale indirizzo metodologico. Ma anche l'approccio costituzionalistico tipico del secondo periodo mostra, in questo momento, i suoi limiti. La presenza di normative sopranazionali direttamente applicabili dal giudice italiano e il peso che, per il diritto interno, vanno assumendo le Corti europee (Strasburgo e Lussemburgo), pongono nuove sfide sul terreno della riflessione teorica. La molteplicità e varietà delle fonti normative complica l'esegesi e rende la ricostruzione sistematica degli istituti assai più impegnativa di quel che questa non fosse quando tutto era regolato dalla legge statale. La classificazione dei principi regolatori del processo penale va riveduta alla luce anche delle norme comunitarie e degli argomenti sviluppati nella giurisprudenza delle Corti europee. Al qual riguardo è utilissima la comparazione con altre realtà (almeno quelle più importanti in Europa), per maturare quella giusta distanza che consenta di guardare dall'esterno il nostro ordinamento processuale. Quella distanza straniante che permetta di coglierne i nessi profondi con visioni culturali, politiche, religiose magari affini eppur diverse dalla nostra, sì da porsi quali condizioni pregiuridiche di soluzioni normative considerate ovvie e irrinunciabili, se percepite nel ristretto orizzonte del diritto interno ${ }^{48}$. Per le stesse ragioni appare

48 La letteratura processualpenalistica italiana sconta un certo ritardo sul terreno delle comparazione sincronica. A parte alcune felici eccezioni (riguardanti soprattutto il raffronto con gli ordinamenti di common law) c'è stata finora scarsa propensione per quei sistemi di giustizia penale stranieri appartenenti alla tradizione di civil law e, quindi, più facilmente comparabili con il nostro. Diversamente, le dottrine del processo civile a partire da Giuseppe Chiovenda e ancor prima con Ludovico Mortara, hanno tratto linfa vitale dalla comparazione: al proposito, si veda la bella e istruttiva sintesi di V. Denti, Diritto comparato e scienza processuale, in Riv. dir. proc. 1979, 334 ss. Oggi quel ritardo va colmato per ragioni non solo culturali, ma anche pratiche. Si pensi all'applicazione del mandato d'arresto europeo, quando si debba stabilire se dal diritto straniero derivino ostacoli alla consegna dell'estradando (esemplare, al riguardo, Cass. s.u. 30 gennaio 2007, n. 4614, Ramoci). E, ancora, si pensi alle sentenze con le quali la Corte di Strasburgo condanna uno Stato straniero per violazioni del fair trial: occorre avere una sufficiente conoscenza dell'ordinamento processuale nel quale la violazione è maturata, 
essenziale anche la comparazione storica, dalla quale possono scaturire preziosi contributi circa lo genesi e l'evolversi degli istituti processuali. Importante, infine, lo studio dei risultati che il sistema di giustizia penale concretamente produce, giacché la bontà di una norma o di un'indirizzo giurisprudenziale non possono essere valutate solo per la loro coerenza con principi astratti, se poi questi vengono disattesi nella quotidianità. La procedura penale è una scienza pratica che deve saper dire la sua circa lopportunità di certe soluzioni tecnico-normative, anche valorizzando i dati quantitativi (ad es. sulla quantità di prescrizioni, o di archiviazioni o di accessi al patteggiamento etc.).

Infine, l'impostazione riformista. Il primo codice dell'Italia repubblicana è nato nell'"età della decodificazione". Una battuta paradossale che però corrisponde alla realtà delle cose. Anche per il diritto processuale - che ben più dei diritti sostanziali aspira a una regolamentazione coerente ed unitaria - la forma "codice" si rivela essere un contenitore stretto, angusto. Lopera portata a termine nel 1988 è ben lontana dall'essere quel modello di completezza duraturo nel tempo rappresentato, ad esempio, dal codice del 1930, rimasto pressoché invariato per circa un quarto di secolo. Questo è il tempo delle incessanti riforme propiziate da una molteplicità di fattori difficilmente controllabili, di carattere sia giuridico (moniti o declaratorie di illegittimità della Corte costituzionale, condanne della Corte di Strasburgo, decisioni della Corte di giustizia UE, decisioni quadro e direttive comunitarie) sia extragiuridico (campagne di stampa portatrici di un allarme sociale che il politico di turno si sforza di sedare mettendo mano alla leva del diritto e del processo penale). Nel tempo che ci separa dal 24 ottobre 1989 si sono registrate numerose revisioni della legge processuale. Quella delle riforme settoriali sembra essere, al momento, l'unica via rimasta per revisionare la legge processuale ${ }^{49}$.

per capire se gli argomenti esposti in quella condanna possano fornire anche al difensore italiano spunti favorevoli al proprio assistito. E rilievi analoghi valgono per le decisioni della Corte di giustizia europea, che risolvano questioni pregiudiziali relative a norme di ordinamenti stranieri.

Due tentativi intrapresi di recente di ricompilare il codice del 1988 sono rimasti senza esito. Nel 2005, la Commissione ministeriale presieduta dal prof. 
L'aspirazione verso un nuovo codice ha bisogno di una nuova utopia capace di misurarsi su tempi lunghi. E quella che stiamo vivendo non è epoca di utopie, ma di bilanci a breve termine che esigono atteggiamenti pragmatici.

Insomma, chi credeva che il nuovo codice, grazie alla sua coerenza sistematica, avrebbe contribuito a stabilizzare le norme regolatrici del processo penale è stato smentito. In realtà, dopo la riforma, cresce l'instabilità normativa ${ }^{50}$ e si accentua altresì l'incertezza sugli scenari futuribili. Comprensibilmente, l'innovazione scioglie la tensione ideale, il senso di utopia, che aveva attraversato gli anni precedenti, almeno a partire dallo "schema Carnelutti". All'euforia del nuovo si contrappone ben presto il timore depressivo dell'ignoto. L'entusiasmo della fase progettuale lascia posto alle reali necessità di far funzionare la "macchina dell giustizia" ${ }^{51}$. La riforma diventa terreno di scontro fra chi intende difenderla e magari completarla in senso garantistico e chi la avversa, perché vi ravvisa un cedimento al delitto, letale per la società. Di qui i molti tentativi, spesso riusciti, di riformare la riforma, con interventi particolaristici, quasi sempre "giustificati" da concrete vicende processuali o da scandali capaci di scuotere l'opinione pubblica.

Nino Dalia ha riscritto il codice di procedura penale, nel tentativo di riordinare e razionalizzare una normativa che s'era andata sfilacciando. L'articolato non è stato però mai fatto oggetto di un'iniziativa legislativa. Analoga sorte ha subito il disegno di legge delega portato a termine dalla Commissione presieduta dal prof. Giuseppe Riccio nel febbraio 2008.

${ }^{50}$ Un giornalista de $\mathrm{Il}$ sole -24 ore (edizione del 30 novembre 2009, p. 9) ha calcolato che, nei suoi primi vent'anni, il codice ha subito complessivamente 1.016 modifiche, 846 per iniziativa del legislatore e 170 a seguito di pronunce della Corte costituzionale.

51 Nell'interessante saggio apparso sui Quaderni fiorentini Ennio Amodio è incline a vedere nella circostanza che la fase post-codicem fu gestita dagli apparati ministeriali la ragione del repentino fallimento del nuovo codice. Diversamente sarebbero andate le cose - secondo Amodio - se gli accademici, i "giuristi", non fossero stati estromessi dal dialogo con le istituzioni nella delicata fase di prima applicazione della riforma (cfr., in particolare, $\mathrm{p}$. 380-383). A me pare, però, che la reazione negativa al nuovo codice venne, all'epoca, più dai magistrati attivi negli uffici giudiziari, che da quelli operanti presso l'ufficio legislativo del ministero della giustizia. 
La lunga sequela di modifiche che ne risulta, priva di un'ideale linea-guida, risulta persino difficile da illustrare. E' tuttavia possibile distinguere alcune fasi nell'evoluzione normativa di questo terzo, tortuoso periodo.

Per comodità espositiva suggerisco una suddivisione in quattro tempi: il "triennio sperimentale" (fino al 1992); "l'epoca di Mani pulite", dal 1992 al 1997; "l'epoca del giusto processo”, dal 1997 al 2001; "l'epoca dell'ossessione securitaria e l'apertura allo spazio giuridico europeo", dal 2001 ai giorni nostri.

a) Il triennio sperimentale (1989-1992). - La legge-delega del 1987 (art. 7) attribuiva al Governo il potere di emanare "norme integrative e correttive, nel rispetto dei principi e criteri direttivi fissati dagli artt. 2 e 3 " entro tre anni dall'entrata in vigore del codice. Consapevole delle difficoltà che l'applicazione della nuova legge processuale avrebbe incontrato nella pratica, il legislatore aveva opportunamente sancito l'ultrattività della legge-delega. Ciò al fine di consentire, per la via rapida del decreto delegato, quelle correzioni o aggiunte, in corso d'opera, che la pratica applicazione della nuova normativa avesse richiesto. Di questa chance il governo profitterà una sola volta, per aggiustamenti numerosi, ma di scarsa importanza ${ }^{52}$. Ben più importanti sono le novità che stanno maturando nel vivo confronto fra i soggetti processuali.

Lentrata in vigore del codice determina - come detto - significativi cambiamenti nei rapporti di forza interni all'accertamento penale. Cambiamenti che si scontrano con prassi e mentalità coltivate e a lungo sedimentate sotto il codice previgente. Ora sono soprattutto i magistrati del pubblico ministero a lamentare l'irragionevolezza del nuovo impianto codicistico. Criticano apertamente la rigida separatezza fra fase preliminare e fase del giudizio. Denunciano l'assurdità di taluni divieti probatori che rendono difficoltoso sostenere con successo l'accusa in giudizio. Il disagio si traduce nella sollecitazione a sollevare questioni di illegittimità davanti alla Corte costituzionale, allo scopo di superare la divisione tra indagine preliminare e dibattimento; una divisione essen-

52 Con il d. lgs. 14 gennaio 1991, n. 12 (Disposizioni integrative e correttive della disciplina processuale penalee delle norme ad essa collegate). 
ziale per l'equilibrio del sistema, perché costituiva l'architrave del nuovo ordinamento processuale ${ }^{53}$. Ben presto, il destino del processo riformato è nelle mani dei giudici costituzionali.

Nel 1992, con tre note sentenze (nr. 24, nr. 254 e nr. 255), la Corte attua una vera controriforma del nostro diritto processuale. Il governo va nella stessa direzione, quando, sull'onda emotiva provocata dalle due stragi palermitane di Capaci e di via D'Amelio, approva un decreto-legge (d.1. n. 306/1992 convertito nella 1. 356/1992) che accresce ulteriormente il peso dell'indagine preliminare nell'economia del giudizio di merito. Ne esce ingigantita la posizione del pubblico ministero, dominus non solo della fase investigativa, ma dell'intero processo. Correlativamente ne esce marginalizzata, per non dire mortificata la posizione del difensore.

b) L'epoca di "Mani pulite" (1992-1997). - Non sapremo mai come sarebbero finiti i processi per fatti di corruzione politica successivi al 1992, se l'accertamento delle responsabilità penali fosse avvenuto secondo le regole originariamente previste nel codice del 1989. Possiamo solo immaginarlo. Certo è che, la segnalata controriforma ha grandemente agevolato la funzione accusatoria del pubblico ministero e, con essa, l'ipoteca della fase preliminare sugli esiti del giudizio. Di efficacia davvero formidabile si rivelò, in particolare, la possibilità - dischiusa da Corte cost. 254/1992 - di usare come prove d'accusa dichiarazioni

53 Viene alla mente un'arguta notazione fatta da A. Malinverni, La riforma del processo penale, cit. p. 45, dove, nel ricordare l'origine delle difficoltà a realizzare un nuovo codice di rito avverte che "nella storia della società umana, più di una volta è accaduto di vedere leggi processuali, ispirate dal principio di autorità, venir applicate da giudici aperti alla dialettica, e leggi processuali ispirate dal principio di libertà, venir applicate da giudici fedeli al principio di autorità". Donde la possibilità che la legge sia interpretata in maniera non corrispondente ai suoi fni e che la sua applicazione sia gravemente ostacolata o distorta. Non sempre, in effetti, (anzi raramente) i tempi della politica coincidono con quelli del costume giudiziario e forense. Le parole appena citate sono seguite da tre pagine dense di sano reallismo e disillusa saggezza sul modo di realizzare le riforme processuali. Parole non molto ascoltate allora. Parole da leggere, rileggere e meditare attentamente ancor oggi, a oltre quarant'anni di distanza. 
raccolte unilateralmente dal pubblico ministero, non verificate nel contraddittorio dibattimentale.

E' altrettanto certo che lo squilibrio fra le parti processuali derivante dal rinnovato contesto normativo fu allorigine di un conflitto patologico fra accusa e difesa: un conflitto fra funzioni che si trasformò in conflitto aperto fra magistrati e avvocati, segnatamente, fra magistrati dell'ANM e avvocati delle Camere penali. Del resto, anche di fronte alla collettività, un simile squilibrio di posizioni poteva essere giustificato solo con il classico argomento del "pericolo sociale", del "nemico da combattere" (il politico corrotto, il mafioso, etc.). Quando il senso del pericolo si attenua o svanisce, quello squilibrio appare inaccettabile, perché persino l'opinione pubblica poco sensibile alle ragioni del garantismo intravede il rischio dell'eccesso di zelo, pronto a degenerare in arbitrio.

c) L'epoca del "giusto processo" (1997-2001). - Il conflitto fra magistratura e avvocatura generatosi durante l'esperienza di Mani pulite ha prodotto due iniziative legislative che meritano di essere qui segnalate: la prima volta a rafforzare la posizione della difesa durante la fase preliminare e, in particolare, nel procedimento cautelare (1.332/1995); l'altra tesa ad affermare il diritto dell'imputato a confrontarsi con i testi d'accusa $(1.267 / 1997)^{54}$.

Questa seconda legge è concepita in aperta polemica con la "controriforma" del 1992. Con essa si pone, di fatto, il problema di un recupero dello spirito che aveva animato la riforma processuale del 1988. La rea-

${ }^{54}$ Desta peraltro sorpresa la circostanza che l'iniziativa legislativa del 1997 si sia limitata alla modifica del solo art. 513. Evidentemente, i politici coinvolti nei processi per fatti di corruzione avvertirono con particolare urgenza la necessità di porre un argine all'uso massiccio di chiamate in correità non riscontrate davanti al giudice del dibattimento. Altrettanto grave era il vulnus al contraddittorio connesso con l'uso di dichiarazioni contestate al teste autoconfutantesi (art. 500) o di testimonianze de relato della polizia giudiziaria su dichiarazioni raccolte durante l'indagine preliminare (art. 195 comma 4). L'iniziativa del 1997 parziale, settoriale, mirata su determinate situazioni processuali, in quanto tale sospetta di configurarsi come legge-provvedimento sarà caratteristica (patologica) di numerose riforme processuali degli anni successivi, marchiate come leggi ad personam. 
zione della magistratura non si fa attendere. Nell'arco di pochi mesi, la 1. 267/1997 subirà una fitta gragnuola di eccezioni d'illegittimità (più di cento). Anche in questo caso, la Corte costituzionale si mostrerà sensibile alle obiezioni dei giudici remittenti ed emetterà una decisione (sent.361/1998) che finirà praticamente col neutralizzare la portata garantista della legge impugnata. Ne scaturisce un conflitto fra Parlamento e Corte costituzionale destinato a sfociare, un anno più tardi nella revisione dell'art. 111 cost., vale a dire nella riforma detta del "giusto processo" (1. cost. 2/1999).

Concepita ufficialmente per conferire rango costitruzionale all'idea di proces equitable (fair trial) delineata nell'art. 6 CEDU, la modifica dell'art. 111 si segnala anche per la puntigliosità con la quale - al comma $4^{\circ}$ - vieta un accertamento di colpevolezza sulla scorta di dichiarazioni rese " $d a$ chi, per libera scelta, si sia sempre volontariamente sottratto all'interrogatorio da parte dell'imputato". Parole nelle quali è ravvisabile una diretta reazione alle regole permissive che, dal 1992, consentivano condanne basate su dichiarazioni accusatorie non ribadite in sede dibattimentale, da chi poteva trasformarle in prova semplicemente avvalendosi del proprio diritto al silenzio. La revisione costituzionale segna un passo significativo verso l'affermazione di un'idea di individuo inedita per la nostra cultura giuridica: titolare non solo del diritto di difendersi, ma altresì del diritto a contribuire direttamente alla formazione del giudizio che lo riguarda, con la propria parola e con la propria volontà ${ }^{55}$.

55 Come osserva P. Costa, Intervento, in questo Volume, il modello adversary decolla in Italia proprio nel momento in cui si va attenuando l'ethos solidaristico che ha ispirato la nostra Costituzione sin dalla sua nascita. Coincidenza non casuale, che merita attenta riflessione. I concetti di individuo e di diritto individuale coltivati dai nostri padri costituenti rinviavano agli ideali egalitari e universalizzanti di matrice illuministica, non privi di venature religiose; supponevano, inoltre, un individuo definito socialmente, del quale lo Stato si prende cura (persino quando lo punisce). In tempi recenti è affiorata però anche sul terreno del processo penale una diversa concezione di individuo, come entità dominata dall'idea del proprio interesse e naturalmente contrapposta allo Stato: un'idea - come nota P. Costa - affine a quella in voga nella cultura giuridica o, meglio, nella "antropologia politica" nordamericana. Codesta evoluzione del concetto di individuo trova conferma, a mio avviso, nella crescente importanza che il "formante giurisprudenziale" sta facendo registrare anche negli ordinamenti di civil law, incluso quello italiano. L'influsso delle 
Seguono due provvedimenti legislativi volti ad attuare, almeno in parte, l'idea di giusto processo penale, ancora una volta attraverso la ridefinizione dei rapporti di forza fra soggetti processuali.

La legge 397/2000 disciplinerà analiticamente le indagini difensive, nell'intento di avvicinare la situazione del patrocinatore delle parti private alla posizione del pubblico ministero.

Subito dopo, la legge 63/2001 tradurrà in norme ordinarie i nuovi principi del giusto processo dedicati al modo di formare la prova dichiarativa (commi 3-5 del citato art. 111), sicché la Corte costituzionale, preso atto dei mutati parametri di costituzionalità, si vedrà costretta a superare la giurisprudenza elaborata nel $1992^{56}$.

d) L'epoca della "ossessione securitaria" e l'apertura allo spazio giuridico europeo (2001-2010). - Il tema dei diritti individuali vive una nuova stagione all'alba del nuovo secolo. La tragedia del settembre 2001 induce pressoché tutti gli stati dell'Occidente a varare leggi speciali per fronteggiare il terrorismo internazionale. Le tecniche d'indagine debbono essere all'altezza delle nuove sfide. La posta in gioco (l'entità dei danni temuti) è a tal punto elevata da far apparire necessario l'approntamento di mezzi eccezionali per prevenire il paventato delitto, più che per reprimerlo. L'attività di contrasto tende a spostarsi sul terreno dell'iniziativa poliziesca e di intelligence, con conseguente attenuazione delle garanzie insite nel controllo giudiziario. La sicurezza dei cittadini esige nuovi sacrifici nel quadro di un nuovo concetto della cooperazione giudiziaria.

Nessun politico è insensibile alle ricadute, in termini di consenso, che le iniziative volte ad accrescere il senso di sicurezza sono in

parti sull'evoluzione della giurisprudenza è un tratto tipico dell'adversarial legalism orientato in senso marcatamente individualistico, come segnala R. A. Kagan, The Adversarial Legalism. The American Way of Law 2001, trad. it. La giustizia americana, Come il contraddittorio fa il diritto, Bologna, 2009, p. 49 e, per quanto concerne il processo penale, p. 157 ss. Credo, inoltre, che molti contrasti dottrinali (ad esempio, in tema di valore oggettivo o soltanto soggettivo del principio del contraddittorio o del principio di ragionevole durata del processo) nascano precisamente dal diverso concetto di individuo che gli autori assumono a premessa dei propri svolgimenti argomentativi.

56 Si vedano, in particolare, sent. 32/2002 e ord. 36/2002. 
grado di assicurare. Il discorso vale anche per la politica europea. Credo, ad esempio, che - senza lo shock dell'11 settembre 2001 - non avremmo mai avuto la decisione-quadro sul mandato d'arresto europeo (2002), né sarebbero state messe a punto altre modalità agevolate di cooperazione fra polizia e magistratura degli stati UE, per un più efficace contrasto del crimine transnazionale.

Il pensiero securitario - trasmodato in vera ossessione anche grazie all'uso spregiudicato dei mezzi di comunicazione sociale - rivela elevate capacità di contaminazione e di diffusione ben al di là del crimine transnazionale. Esso nutre modelli di trattamento ispirati a un diritto penale della prevenzione, caratterizzato - sul terreno penale sostanziale - da una marcata anticipazione delle soglie di punibilità e - sul terreno processuale - da un correlativo uso degli strumenti cautelari in funzione di neutralizzazione della pericolosità.

Ne risulta uno spostamento del confine fra prevenzione e repressione, sul quale sono state calibrate le tradizionali categorie del garantismo penale e della tutela dei diritti individuali ${ }^{57}$. Abbondanti esempi sono riscontrabili nella legislazione (non solo italiana) in tema di immigrazione clandestina, di sicurezza del lavoro, di circolazione stradale, di inquinamento ambientale, di violenze domestiche e nei rapporti fra $\mathrm{i}$ sessi. Si pensi ancora alle molte modifiche intervenute sulla disciplina del sequestro e della confisca nel procedimento di prevenzione ante-delictum (1.575/1965). E lo stesso codice di rito offre qualche esempio che dimostra l'inclinazione preventiva del sistema processuale: si pensi ai numerosi interventi novellistici sull'art. 275 comma 3, in tema di adeguatezza presunta della custodia cautelare in carcere per reati di allarme sociale.

La politica securitaria tende ad attuarsi per la via diretta del diritto amministrativo e di polizia, proprio mentre si affermano i principi del giusto processo ${ }^{58}$. Sembra una contraddizione, ma non lo è, perché diverse sono le tipologie soggettive pensate come destinatarie dell'uno o

57 Illuminanti, al riguardo, le riflessioni di W. Hassemer, Sicherheit durch Strafrecht, in Strafverteidiger 2006, 322 ss. e, più recentemente, in Warum Strafe sein muß, Ullstein Buchverlage GmbH, Berlin 2009, soprattutto alle pagine 70 ss.

58 Così, acutamente, P. Costa, Intervento, in questo Volume. 
dell'altro trattamento. Affiora qui un'evidente logica selettiva, non dissimile da quella auspicata dai teorici del Feindstracrecht ${ }^{59}$.

Nel nuovo contesto politico-sociale, i diritti individuali a rischio sono principalmente quelli minacciati da iniziative preventive confidate alle agenzie di controllo sociale (polizia e pubblici ministeri): iniziative spesso di carattere amministrativo, sfornite pertanto del corredo di garanzie assicurate per il procedimento penale (oltre al procedimento di prevenzione ante delictum, il pensiero va alle procedure di controllo sull'immigrazione clandestina regolate dal d.lgs 286/1998, ripetutamente modificato dai vari "pacchetti sicurezza").

Il diritto penale della sicurezza apre una nuova stagione dei diritti individuali, per la cui tutela si rivela sempre più importante il ruolo della Corte europea dei diritti dell'uomo. Pur guardata con sospetto da parte della dottrina processualistica ${ }^{60}$, la Corte di Strasburgo ha sviluppato nel tempo una giurisprudenza attenta alla "sostanza" dei diritti individuali. La circostanza poi che la Corte EDU guardi al concreto comportamento di volta in volta tenuto dall'autorità pubblica attribuisce alle sue decisioni un carattere di duttilità ignoto alla sentenze della nostra Corte costituzionale. Pur consapevole dei rischi insiti nell'affidarsi al responso di giurisdizioni sovrastatuali, penso dunque vada salutato con favore il riconoscimento delle nostre alte Corti (di cassazione e costituzionale) rispetto sia all'effetto delle sentenze europee sui giudicati interni ${ }^{61}$, sia al

59 La nota tesi di G. Jakobs, secondo la quale occorre creare un diritto penale volto alla immediata neutralizzazione di condotte pericolose per il contesto sociale, quali quelle di terroristi, pedofili, delinquenti abituali (Feindstrafrecht), al fine di far salve le tradizionali garanzie del diritto penale per tutti gli altri cittadini (Bürgerstrafrecht). Per una succinta esposizione delle idee di Jakobs si veda, in particolare, il saggio I terroristi non hanno diritti, in Contrasto al terrorismo interno e internazionale, a cura di R. Kostoris e R. Orlandi, p. 3 ss.

${ }^{60}$ Critica la tendenza (giurispridenziale e dottrinale) a ragioni del decidere espresse nelle motivazioni delle sentenze della Corte di Strasburgo P. Ferrua, Il giudizio penale: fatto e valore giuridico, in P. Ferrua, F.M. Grifantini, G. Illuminati, R. Orlandi, La prova nel dibattimento penale, $4^{\circ}$ ed., Torino 2010, p.406 ss.

${ }^{61}$ Si vedano le sentenze emesse nei noti casi Sejdovic (2006), Dorigo (2007) e Drassich (2009) con le quali si riconosce che la condanna dello Stato italiano da parte della Corte EDU sia idonea a risolvere l'efficacia del giudicato interno. 
rango "para-costituzionale" delle norme convenzionali e dei principi in esse affermati ${ }^{62}$. Tale riconoscimento segnala, al contempo, un diverso sguardo alla tematica dei diritti individuali. Alla lunga, gli argomenti della Corte di Strasburgo sui limiti di tutela della persona (argomenti orientati al principio di proporzionalità più che al criterio di ragionevolezza) finiranno col contaminare anche la giurisprudenza e la dottrina italiane.

6. Conclusioni. - E' ora di tirare le fila al termine del lungo itinerario. Poche battute saranno sufficienti a svelarne il senso.

Caduto il fascismo, con l'Italia ancora in guerra, si avverte subito l'esigenza di purgare l'ordinamento processuale degli elementi autoritari tipici del passato regime. Lorientamento individualistico della Costituzione repubblicana accentua la necessità della riforma, inizialmente perseguita nell'illusione che sia sufficiente una revisione parziale del codice Rocco.

Al centro degli sforzi riformistici è principalmente il diritto di difesa, del quale si reclama l'attuazione "in ogni stato e grado del procedimento", come scritto nell'art. 24 comma 2 cost. L'enfasi sulla garanzia difensiva sposta ben presto l'asse dell'interesse (e delle discussioni) sui rapporti di forza interni al processo. L'attuazione dei diritti individuali diventa così una funzione della dialettica accusa/difesa e della scelta fra stile inquisitorio e modello accusatorio.

Di qui il carattere fortemente ideologico con tinte, per così dire, corporative che le dispute sulla riforma processuale assumono nel dibattito italiano. Soprattutto a partire dall'inizio degli anni '60, con la svolta segnata dall'iniziativa di Carnelutti, il tema dei diritti individuali è per così dire assorbito e confuso nella disputa fra accusatorio e inquisitorio. Attuare il processo di parti, è la via maestra per salvaguardare al contempo i diritti individuali minacciati o compressi dall'attività giudiziaria e di polizia.

62 Si vedano le sent. 348 e 349/2007 che - facendo leva sull'art. 117 comma 1 cost. - attribuiscono ai principi convenzionali il rango di "norme interposte", suscettibili di fungere da parametro per il giudizio di legittimità della legge italiana. Va peraltro segnalato che, a partire dal $1^{\circ}$ dicembre 2009 , le norme della convenzione europea dei diritti dell'uomo sono divenute parte integrante del diritto comunitario, grazie al loro recepimento nell'art. 6 del Trattato di Lisbona (come riconosce la stessa Corte cost. nella sent. 138/2010). 
Negli anni'60 e'70, la Corte costituzionale si muove nella stessa direzione, sviluppando a sua volta una giurisprudenza processuale penale incentrata principalmente intorno all'art. 24 comma 2, spesso combinato con l'art. 3 cost. Le questioni di legittimità vengono esaminate alla luce del criterio (marcatamente politico) della ragionevolezza, anziché in base a quello (più connotato in senso giuridico) della proporzionalità fra diritto violato e scopo perseguito dalla misura restrittiva ${ }^{63}$.

L'iniziale fallimento della riforma processuale entrata in vigore nel 1989 ha accentuato il contrasto fra i soggetti processuali. I conflitti e le polemiche seguiti all'esperienza di Mani pulite hanno trovato una composizione momentanea nella riforma costituzionale del "giusto processo" (1999). I motivi di tensione non sono peraltro venuti meno, come dimostrano le perduranti incomprensioni fra avvocatura e magistratura, alle quali si è aggiunta, nell'ultimo decennio, la difficile convivenza fra magistratura e classe politica di governo. I toni accesi e spesso personalistici delle dispute non devono trarre in inganno. Non si tratta di polemiche destinate a sparire con i protagonisti del momento. E' in gioco qualcosa di più serio e importante che tocca un presupposto essenziale della cultura giuridica: il concetto di individuo al centro di una contesa ancora irrisolta fra ideale illuministico e approccio pragmatico-utilitaristico.

L'evoluzione del sistema penale in senso preventivo (soprattutto a partire dal 2001) e il diffondersi di un'ideologia securitaria, esigono una rivisitazione, un aggiornamento delle classiche categorie del garantismo, maturate sul presupposto di una chiara (almeno in teoria) separazione tra prevenzione e repressione. Al contempo, lo sviluppo di modalità investigative che sfruttano l'uso di moderne risorse tecnologiche apre prospettive inedite per diritti individuali non riducibili al diritto di difesa e, forse per questo, rimasti fino ad ora ai margini dell'attenzione del processualpenalista: penso al diritto della riservatezza, del quale si fatica a trovare un ancoramento costituzionale.

${ }^{63}$ Questo è invece, come già ricordato, il criterio preferito dalla Corte costituzionale tedesca, già a partire dai primi anni ' 50 . Un criterio - val la pena aggiungere - prossimo all'ideale egalitario e universalizzante di provenienza illuministica più che all'approccio pragmatico-utilitaristico tipico della cultura giuridica americana. 
L'apertura dell'Italia allo spazio giuridico europeo e i nuovi scenari della cooperazione giudiziaria internazionale pongono nuove sfide sul terreno dei diritti individuali. La loro tutela trova spesso risposte adeguate nella giurisprudenza della Corte di Strasburgo, capace di fungere da modello (soprattutto quanto a modalità argomentative) anche per i giudici italiani.

\section{COMO CITAR ESTE ARTIGO:}

ORLANDI, Renzo. Diritti individuali e processo penale nell'Italia repubblicana. Revista Brasileira de Direito Processual Penal, Porto Alegre, vol. 2, n. 1, p. 7 - 41, 2016. http://dx.doi.org/10.22197/rbdpp.v2i1.15 